COMMUNICATIONS IN

ANALYSIS AND GEOMETRY

Volume 15, Number 1, 175-194, 2007

\title{
A general gap theorem for submanifolds with parallel mean curvature in $R^{n+p}$
}

\author{
HONG-WEI Xu AND JUAN-RU Gu
}

Let $M^{n}(n \geq 3)$ be an $n$-dimensional complete submanifold with parallel mean curvature in $\mathbf{R}^{n+p}$. Denote by $H$ and $S$ the mean curvature and the squared length of the second fundamental form of $M$, respectively. By using a direct method, we prove that if $\int_{M}\left(S-n H^{2}\right)^{n / 2} d M<C(n)$, where $C(n)$ is an explicit positive constant depending only on $n$, then $S \equiv n H^{2}$, i.e., $M^{n}$ is a totally umbilical submanifold. In particular, if $H=0$, then $M=\mathbf{R}^{n}$; if $H \neq 0$, then $M=S^{n}(1 / H)$. It improves the gap theorems proposed by Lei $\mathrm{Ni}$ and $\mathrm{Xu}$.

\section{Introduction}

An important problem in global differential geometry is the study of relations between geometrical invariants and structures of Riemannian manifolds or submanifolds. After the pioneering rigidity theorem for closed minimal submanifolds in a sphere proposed by Simons [12], Lawson [5], Chern et al. [2] and $\mathrm{Li}$ and $\mathrm{Li}$ [6] improved the Simons' pinching constant for $n$ dimensional closed minimal submanifolds in $S^{n+p}(1)$ to $\max \left\{\frac{n}{2-1 / p}, \frac{2}{3} n\right\}$, which is optimal for minimal surfaces and minimal hypersurfaces in a sphere. The following result, known as rigidity theorem for submanifolds with parallel mean curvature in a sphere, was first proved by Okumura [10], Yau [22], and later by $\mathrm{Xu}$ [17] and finally by Alencar-do Carmo [1] for codimension 1 case and $\mathrm{Xu}[18]$ for codimension $p$ case independently, as stated

Theorem 1.1. For a given constant $H \geq 0$ and positive integers $n(\geq 2)$, $p$, there exists a positive number $C_{1}(n, p, H)$ with the following property: if $M^{n}$ is an $n$-dimensional closed submanifold in an $(n+p)$-dimensional unit sphere $S^{n+p}(1)$ with parallel mean curvature vector having norm $H$, and if 
$S$ is the squared norm of the second fundamental form satisfying

$$
S \leq C_{1}(n, p, H)
$$

then $M$ is congruent to one of the following:

(i) $S^{n}\left(\frac{1}{\sqrt{1+H^{2}}}\right)$;

(ii) the isoparametric hypersurface $S^{n-1}\left(\frac{1}{\sqrt{1+\lambda^{2}}}\right) \times S^{1}\left(\frac{\lambda}{\sqrt{1+\lambda^{2}}}\right)$ in $S^{n+1}(1)$;

(iii) one of the Clifford minimal hypersurfaces $S^{k}\left(\sqrt{\frac{k}{n}}\right) \times S^{n-k}\left(\sqrt{\frac{n-k}{n}}\right)$ in $S^{n+1}(1)$ for $k=1, \ldots, n-1$;

(iv) the Clifford torus $S^{1}\left(r_{1}\right) \times S^{1}\left(r_{2}\right)$ in $S^{3}(r)$ with constant mean curvature $H_{0}$, where $r_{1}, r_{2}=\left[2\left(1+H^{2}\right) \pm 2 H_{0}\left(1+H^{2}\right)^{1 / 2}\right]^{-1 / 2}, r=(1+$ $\left.H^{2}-H_{0}^{2}\right)^{-1 / 2}$ and $0 \leq H_{0} \leq H$

(v) the Veronese surface in $S^{4}\left(\frac{1}{\sqrt{1+H^{2}}}\right)$.

Here $\lambda$ and $C_{1}(n, p, H)$ are given by

$$
\lambda=\frac{n H+\sqrt{n^{2} H^{2}+4(n-1)}}{2(n-1)}
$$

and

$$
\begin{aligned}
& C_{1}(n, p, H) \\
& \quad= \begin{cases}A(n, H), & \text { for } p=1, \text { or } p=2 \text { and } H \neq 0, \\
\min \left\{A(n, H), \frac{1}{3}\left(2 n+5 n H^{2}\right)\right\}, & \text { for } p \geq 3, \text { or } p=2 \text { and } H=0,\end{cases}
\end{aligned}
$$

where

$$
A(n, H)=n+\frac{n^{3} H^{2}}{2(n-1)}-\frac{n(n-2) H}{2(n-1)} \sqrt{n^{2} H^{2}+4(n-1)} .
$$

In particular, if $H \neq 0$, and if

$$
S \leq C_{2}(n, p),
$$

then $M$ is either a totally umbilical sphere in $S^{n+p}(1)$, a Clifford isoparametric hypersurface in an $(n+1)$-dimensional sphere, or the Veronese surface 
in $S^{4}\left(\frac{1}{\sqrt{1+H^{2}}}\right)$. Here

$$
C_{2}(n, p)= \begin{cases}2 \sqrt{n-1}, & \text { for } p \leq 2, \text { or } n \geq 8 \text { and } p \geq 3, \\ \frac{2}{3} n, & \text { for } n \leq 7 \text { and } p \geq 3 .\end{cases}
$$

Further discussions in this direction have been carried out by Shiohama and $\mathrm{Xu}[21,13,14]$. However, all these results have pointwise condition for $S$. It seems to be interesting to study rigidity for minimal submanifolds under $L^{n / 2}$-pinching condition for $S$. The $L^{n / 2}$-pinching theorem for minimal submanifolds in a sphere was initiated by Shen [11], and later investigated by Wang [16], Lin and Xia [8] and Xu [19]. In [20], Xu proved the following theorem.

Theorem 1.2. Let $M^{n}$ be an n-dimensional closed submanifold with parallel mean curvature in $S^{n+p}(1)$. If $\int_{M}\left(S-n H^{2}\right)^{n / 2} d M<C_{3}(n)$, where $C_{3}(n)$ is an explicit positive constant depending only on $n$, then $M$ is a totally umbilical sphere.

The total curvature $\int_{M}\left(S-n H^{2}\right)^{n / 2} d M$ is also called Willmore functional [3]. In the case where $M$ is a complete minimal hypersurface in $\mathbf{R}^{n+1}$, $\mathrm{Ni}[9]$ obtained the following theorem.

Theorem 1.3. Let $M^{n}(n \geq 4)$ be an $n$-dimensional complete minimal hypersurface in $\mathbf{R}^{n+1}$. Then, there exists an explicit positive constant $C_{4}(n)$ depending only on $n$ such that if $\int_{M} S^{n / 2} d M<C_{4}(n)$, M must be a totally geodesic hyperplane.

In the present article, we mainly study the $L^{n / 2}$-pinching problem for $n$-dimensional complete submanifolds with parallel mean curvature in the Euclidean space $\mathbf{R}^{n+p}$. By using a direct method, we obtain the following gap theorem.

Theorem 1.4. Let $M^{n}(n \geq 3)$ be an $n$-dimensional complete submanifold with parallel mean curvature in $\mathbf{R}^{n+p}$. Denote by $H$ and $S$ the mean curvature and the squared length of the second fundamental form of $M$, respectively. If $\int_{M}\left(S-n H^{2}\right)^{n / 2} d M<C(n)$, where $C(n)$ is an explicit positive constant depending only on $n$, then $S \equiv n H^{2}$, i.e., $M^{n}$ is a totally umbilical submanifold. In particular, if $H=0$, then $M=\mathbf{R}^{n}$; if $H \neq 0$, then $M=S^{n}(1 / H)$. 
Consequently, we have the following corollary.

Corollary 1.5. Let $M^{n}(n \geq 3)$ be an $n$-dimensional complete minimal submanifold in $\mathbf{R}^{n+p}$. Then there exists an explicit positive constant $C(n)$ depending only on $n$ such that if $\int_{M} S^{n / 2} d M<C(n)$, M must be a totally geodesic submanifold $\mathbf{R}^{n}$.

More general, we obtain the following theorem.

Theorem 1.6. Let $M^{n}(n \geq 3)$ be an $n$-dimensional complete submanifold with parallel mean curvature in $\mathbf{F}^{n+p}(c)$, where $\mathbf{F}^{n+p}(c)$ is an $(n+p)$ dimensional complete simply connected space form with non-negative constant curvature $c$. Denote by $H$ and $S$ the mean curvature and the squared length of the second fundamental form of $M$, respectively. If $\int_{M}(S-$ $\left.n H^{2}\right)^{n / 2} d M<C(n)$, where $C(n)$ is an explicit positive constant depending only on $n$, then $S \equiv n H^{2}$, i.e., $M^{n}$ is a totally umbilical submanifold. In particular, if $c+H^{2}=0$, then $M=\mathbf{R}^{n}$; if $c+H^{2} \neq 0$, then $M=S^{n}\left(\frac{1}{\sqrt{c+H^{2}}}\right)$.

Remark 1.7. When $c=1$ and $M$ is compact, Theorem 1.6 reduces to Theorem 1.2. When $c=0, H=0, p=1$ and $n \geq 4$, Theorem 1.6 reduces to Theorem 1.3. In fact, one can see from the proof of Lemma 3.3 that Theorem 1.4 is equivalent to Theorem 1.6.

\section{Notation and lemmas}

Let $M^{n}$ be an $n$-dimensional Riemannian submanifold immersed in an $(n+p)$-dimensional Euclidean space $\mathbf{R}^{n+p}$. We shall make use of the following convention on the range of indices:

$$
1 \leq A, B, C, \ldots \leq n+p ; \quad 1 \leq i, j, k, \ldots \leq n ; \quad n+1 \leq \alpha, \beta, \gamma, \ldots \leq n+p .
$$

Choose a local orthonormal frame field $\left\{e_{A}\right\}$ in $\mathbf{R}^{n+p}$ such that, restricted to $M$, the $e_{i}$ 's are tangent to $M$. Let $\left\{\omega_{A}\right\}$ and $\left\{\omega_{A B}\right\}$ be the dual frame field and the connection 1 -forms of $\mathbf{R}^{n+p}$, respectively. Restricting these forms to $M$, we have

$$
\begin{aligned}
\omega_{\alpha i} & =\sum_{j} h_{i j}^{\alpha} \omega_{j}, h_{i j}^{\alpha}=h_{j i}^{\alpha}, \\
R_{i j k l} & =\sum_{\alpha}\left(h_{i k}^{\alpha} h_{j l}^{\alpha}-h_{i l}^{\alpha} h_{j k}^{\alpha}\right),
\end{aligned}
$$




$$
\begin{aligned}
R_{\alpha \beta k l} & =\sum_{i}\left(h_{i k}^{\alpha} h_{i l}^{\beta}-h_{i l}^{\alpha} h_{i k}^{\beta}\right), \\
h & =\sum_{\alpha, i, j} h_{i j}^{\alpha} \omega_{i} \otimes \omega_{j} \otimes e_{\alpha}, \\
\xi & =\frac{1}{n} \sum_{\alpha, i} h_{i i}^{\alpha} e_{\alpha},
\end{aligned}
$$

where $R_{i j k l}, R_{\alpha \beta k l}, h$ and $\xi$ are the curvature tensor, the normal curvature tensor, the second fundamental form and the mean curvature vector of $M$, respectively.

We define

$$
S=|h|^{2}, \quad H=|\xi|, \quad H_{\alpha}=\left(h_{i j}^{\alpha}\right)_{n \times n}
$$

Definition 2.1. $M$ is called a submanifold with parallel mean curvature if $\xi$ is parallel in the normal bundle of $M$. In particular, $M$ is called minimal if $\xi=0$.

When $\xi \neq 0$, we choose $e_{n+1}$ such that $e_{n+1} \| \xi$, tr $H_{n+1}=n H$ and $\operatorname{tr} H_{\beta}=0, n+2 \leq \beta \leq n+p$. Set

$$
S_{H}=\sum_{i, j}\left(h_{i j}^{n+1}\right)^{2}, \quad S_{I}=\sum_{i, j, \beta \neq n+1}\left(h_{i j}^{\beta}\right)^{2}
$$

The following lemmas will be used in the proof of our main results.

Lemma 2.2. If $M^{n}$ is a submanifold with parallel mean curvature in a space form of constant curvature, then either $H=0$, or $H$ is constant and $H_{n+1} H_{\alpha}=H_{\alpha} H_{n+1}$, for all $\alpha$.

Lemma $2.3[6,19]$. Let $M^{n}$ be a submanifold with parallel mean curvature in $\mathbf{R}^{n+p}$.

(i) If $H=0$, then

$$
\frac{1}{2} \Delta S \geq \sum_{\alpha, i, j, k}\left(h_{i j k}^{\alpha}\right)^{2}-\mu(p) S^{2}
$$


(ii) If $H \neq 0$, then

$$
\begin{aligned}
\frac{1}{2} \Delta S_{H} \geq & \sum_{i, j, k}\left(h_{i j k}^{n+1}\right)^{2} \\
& +\left(S_{H}-n H^{2}\right)\left[2 n H^{2}-S-\frac{n(n-2) H}{\sqrt{n(n-1)}} \sqrt{S-n H^{2}}\right] \\
\frac{1}{2} \Delta S_{I} \geq & \sum_{i, j, k, \beta \neq n+1}\left(h_{i j k}^{\beta}\right)^{2}+n H \sum_{\beta \neq n+1} \operatorname{tr}\left(H_{n+1} H_{\beta}^{2}\right) \\
& -\sum_{\beta \neq n+1}\left[\operatorname{tr}\left(H_{n+1} H_{\beta}\right)\right]^{2}-\mu(p-1) S_{I}^{2} \quad \text { for } p \neq 1 .
\end{aligned}
$$

Here

$$
\mu(m)= \begin{cases}1 & \text { for } m=1 \\ \frac{3}{2} & \text { for } m \geq 2\end{cases}
$$

By using the same argument as in [20], we have the following lemma.

Lemma 2.4. Let $M^{n}$ be a submanifold with parallel mean curvature in $\mathbf{R}^{n+p}$. Set $f_{\varepsilon}=\left(S_{H}-n H^{2}+n \varepsilon^{2}\right)^{1 / 2}, g_{\varepsilon}=\left[S_{I}+n(p-1) \varepsilon^{2}\right]^{1 / 2}, h_{\varepsilon}=(S+$ $\left.n p \varepsilon^{2}\right)^{1 / 2}$. Then

(i) if $H \neq 0$, then

$$
\begin{aligned}
\sum_{i, j, k}\left(h_{i j k}^{n+1}\right)^{2} & \geq \frac{n+2}{n}\left|\nabla f_{\varepsilon}\right|^{2}, \\
\sum_{i, j, k, \beta \neq n+1}\left(h_{i j k}^{\beta}\right)^{2} & \geq \frac{n+2}{n}\left|\nabla g_{\varepsilon}\right|^{2} \quad \text { for } p \neq 1 .
\end{aligned}
$$

(ii) If $H=0$, then

$$
\sum_{\alpha, i, j, k}\left(h_{i j k}^{\alpha}\right)^{2} \geq \frac{n+2}{n}\left|\nabla h_{\varepsilon}\right|^{2} .
$$

From $[4,20]$, we have the following lemma.

Lemma 2.5. Let $M^{n}(n \geq 3)$ be a compact submanifold with or without boundary with parallel mean curvature in $\mathbf{R}^{n+p}$. Then for all $t \in \mathbf{R}^{+}$, and 
$f \in C^{1}(M), f \geq 0$ (if the boundary $\partial M \neq \emptyset,\left.f\right|_{\partial M}=0$ ), $f$ satisfies

$$
\|\nabla f\|_{2}^{2} \geq \frac{(n-2)^{2}}{4(n-1)^{2}(1+t)}\left[\frac{1}{D^{2}(n)}\|f\|_{2 n /(n-2)}^{2}-H^{2}\left(1+\frac{1}{t}\right)\|f\|_{2}^{2}\right],
$$

where $D(n)=2^{n}(1+n)^{(n+1) / n}(n-1)^{-1} \sigma_{n}^{-1 / n}$ and $\sigma_{n}=$ volume of the unit ball in $\mathbf{R}^{n}$.

\section{Proof of theorems}

We first define our pinching constants as follows

$$
\begin{gathered}
B(n, p, H)= \begin{cases}\alpha(n) & \text { for } p=1 \text { and } H \neq 0, \\
\min \{\alpha(n), \beta(n)\} & \text { for } p=2 \text { and } H \neq 0, \\
\min \left\{\alpha(n), \frac{2}{3} \beta(n)\right\} & \text { for } p \geq 3 \text { and } H \neq 0, \\
\gamma(n) & \text { for } p=1 \text { and } H=0, \\
\frac{2}{3} \gamma(n) & \text { for } p \geq 2 \text { and } H=0,\end{cases} \\
C(n)=\min \left\{\alpha^{n / 2}(n),\left[\frac{2}{3} \beta(n)\right]^{n / 2},\left[\frac{2}{3} \gamma(n)\right]^{n / 2}\right\}
\end{gathered}
$$

where

$$
\begin{aligned}
\alpha(n) & =2 n a_{n} D^{-2}(n)\left[\left(a_{n} b_{n}\right)^{1 / 2}+\left(1+a_{n}\right)^{1 / 2}\left(2+b_{n}\right)^{1 / 2}\right]^{-2}, \\
a_{n} & =\left(n^{2}-n+2\right)(n-2)^{2}\left[n^{4}(n-1)^{2}\right]^{-1}, \quad b_{n}=(n-2)^{2}(2 n-2)^{-1}, \\
\beta(n) & =n\left(n^{2}-n+2\right)(n-2)^{2} D^{-2}(n)\left[n^{4}(n-1)^{2}+\left(n^{2}-n+2\right)(n-2)^{2}\right]^{-1}, \\
\gamma(n) & =\left(n^{2}-n+2\right)(n-2)^{2} D^{-2}(n)\left[n^{3}(n-1)^{2}\right]^{-1} .
\end{aligned}
$$

To prove Theorem 1.4, we give the following key lemma.

Lemma 3.1. Let $M^{n}(n \geq 3)$ be a complete submanifold with parallel mean curvature in $\mathbf{R}^{n+p}$. Suppose that $H \neq 0$ and $\left\|S-n H^{2}\right\|_{n / 2}<\alpha(n)$. Then $M$ is a pseudo-umbilical submanifold. In particular, if $p=1$, then $M$ is a hypersphere in $\mathbf{R}^{n+1}$. Here $\left\|S-n H^{2}\right\|_{K}=\left[\int_{M}\left(S-n H^{2}\right)^{K} d M\right]^{1 / K}$. 
Proof. Putting $f_{\varepsilon}=\left(S_{H}-n H^{2}+n \varepsilon^{2}\right)^{1 / 2}, \quad f=\left(S_{H}-n H^{2}\right)^{1 / 2}$, we have $\Delta f_{\varepsilon}^{2}=\Delta f^{2}$. By Lemmas 2.3 and 2.4 , we obtain

$$
\frac{1}{2} \Delta f_{\varepsilon}^{2} \geq \frac{n+2}{n}\left|\nabla f_{\varepsilon}\right|^{2}+f^{2}\left[2 n H^{2}-S-\frac{n(n-2) H}{\sqrt{n(n-1)}} \sqrt{S-n H^{2}}\right]
$$

We choose a cut-off function $\phi_{R} \in C^{\infty}(M)$ such that

$$
\phi_{R}(x)= \begin{cases}1, & \text { if } x \in B_{R}(q), \\ 0, & \text { if } x \in M \backslash B_{2 R}(q), \\ \phi_{R}(x) \in[0,1] \text { and }\left|\nabla \phi_{R}\right| \leq \frac{1}{R}, & \text { if } x \in B_{2 R}(q) \backslash B_{R}(q),\end{cases}
$$

where $B_{r}(q)$ is the geodesic ball in $M$ with radius $r$ centered at $q \in M$. In particular, if $M$ is compact, and if $R \geq d$, where $d$ is the diameter of $M$, then $\phi_{R} \equiv 1$ on $M$. Multiplying $\phi_{R}^{2} f_{\varepsilon}^{2 k-2}(k \geq 1)$ on both sides of inequality (3.2) and integrating by parts, we get

$$
\begin{aligned}
0 \geq & \int_{M} \phi_{R}^{2} f_{\varepsilon}^{2 k-2} f^{2}\left[2 n H^{2}-S-\frac{n(n-2) H}{\sqrt{n(n-1)}} \sqrt{S-n H^{2}}\right] d M \\
& +\frac{n+2}{n k^{2}} \int_{M} \phi_{R}^{2}\left|\nabla f_{\varepsilon}^{k}\right|^{2} d M \\
& +\frac{1}{2} \int_{M} \nabla\left(\phi_{R}^{2} f_{\varepsilon}^{2 k-2}\right) \nabla f_{\varepsilon}^{2} d M-\frac{1}{2} \int_{M} \operatorname{div}\left(\phi_{R}^{2} f_{\varepsilon}^{2 k-2} \nabla f_{\varepsilon}^{2}\right) d M \\
= & \int_{M} \phi_{R}^{2} f_{\varepsilon}^{2 k-2} f^{2}\left[2 n H^{2}-S-\frac{n(n-2) H}{\sqrt{n(n-1)}} \sqrt{S-n H^{2}}\right] d M \\
& +\frac{2 n k-n+2}{n k^{2}} \int_{M} \phi_{R}^{2}\left|\nabla f_{\varepsilon}^{k}\right|^{2} d M+2 \int_{M} \phi_{R} f_{\varepsilon}^{2 k-1} \nabla \phi_{R} \nabla f_{\varepsilon} d M \\
\geq & \int_{M} \phi_{R}^{2} f_{\varepsilon}^{2 k-2} f^{2}\left[2 n H^{2}-S-\frac{n(n-2) H}{\sqrt{n(n-1)}} \sqrt{S-n H^{2}}\right] d M \\
& +\frac{2 n k-n+2}{n k^{2}} \int_{M} \phi_{R}^{2}\left|\nabla f_{\varepsilon}^{k}\right|^{2} d M+2 \int_{M} \phi_{R} f_{\varepsilon}^{2 k-1} \nabla \phi_{R} \nabla f_{\varepsilon} d M \\
& +\sigma \int_{M} \phi_{R} f_{\varepsilon}^{2 k-1} \nabla \phi_{R} \nabla f_{\varepsilon} d M-\frac{\rho \sigma}{2} \int_{M} \phi_{R}^{2} f_{\varepsilon}^{2 k-2}\left|\nabla f_{\varepsilon}\right|^{2} d M \\
& -\frac{\sigma}{2 \rho} \int_{M}^{\left|\nabla \phi_{R}\right|^{2} f_{\varepsilon}^{2 k} d M}
\end{aligned}
$$


for all $\rho, \sigma \in \mathbf{R}^{+}$. Taking $k=\frac{n}{2}, \sigma=\frac{4\left(n^{2}-n+2\right)}{n^{2}}-\frac{n}{R}-2$ and $\rho=\frac{n^{2}}{2 R \sigma}$, where $R>\frac{n^{3}}{2\left(n^{2}-2 n+4\right)}$, we get

$$
\begin{aligned}
0 \geq & \int_{M} \phi_{R}^{2} f_{\varepsilon}^{n-2} f^{2}\left[2 n H^{2}-S-\frac{n(n-2) H}{\sqrt{n(n-1)}} \sqrt{S-n H^{2}}\right] d M \\
& -\frac{R \sigma^{2}}{n^{2}} \int_{M} f_{\varepsilon}^{n}\left|\nabla \phi_{R}\right|^{2} d M \\
& +\left[\frac{4\left(n^{2}-n+2\right)}{n^{3}}-\frac{1}{R}\right]\left(\int_{M} \phi_{R}^{2}\left|\nabla f_{\varepsilon}^{n / 2}\right|^{2} d M\right. \\
& \left.+n \int_{M} \phi_{R} f_{\varepsilon}^{n-1} \nabla \phi_{R} \nabla f_{\varepsilon} d M\right) \\
\geq & -\left[\frac{4\left(n^{2}-n+2\right)}{n^{3}}-\frac{1}{R}+\frac{R \sigma^{2}}{n^{2}}\right] \int_{M} f_{\varepsilon}^{n}\left|\nabla \phi_{R}\right|^{2} d M \\
& +\left[\frac{4\left(n^{2}-n+2\right)}{n^{3}}-\frac{1}{R}\right] \int_{M}\left|\nabla\left(\phi_{R} f_{\varepsilon}^{n / 2}\right)\right|^{2} d M \\
& +\int_{M} \phi_{R}^{2} f_{\varepsilon}^{n-2} f^{2}\left[2 n H^{2}-S-\frac{n(n-2) H}{\sqrt{n(n-1)}} \sqrt{S-n H^{2}}\right] d M .
\end{aligned}
$$

By Lemma 2.5, we have

$$
\begin{aligned}
\left\|\nabla\left(\phi_{R} f_{\varepsilon}^{n / 2}\right)\right\|_{2}^{2} \geq & \frac{(n-2)^{2}}{4(n-1)^{2}(1+t)}\left[\frac{1}{D^{2}(n)}\left\|\phi_{R} f_{\varepsilon}^{n / 2}\right\|_{2 n /(n-2)}^{2}\right. \\
& \left.-H^{2}\left(1+\frac{1}{t}\right)\left\|\phi_{R} f_{\varepsilon}^{n / 2}\right\|_{2}^{2}\right]
\end{aligned}
$$

for all $t \in \mathbf{R}^{+}$. From (3.4) and (3.5), we obtain

$$
\begin{aligned}
0 \geq & -\left[\frac{4\left(n^{2}-n+2\right)}{n^{3}}-\frac{1}{R}+\frac{R \sigma^{2}}{n^{2}}\right] \int_{M} f_{\varepsilon}^{n}\left|\nabla \phi_{R}\right|^{2} d M \\
& +\frac{\left[4 R\left(n^{2}-n+2\right)-n^{3}\right](n-2)^{2}}{4 R n^{3}(n-1)^{2}(1+t)}\left[\frac{1}{D^{2}(n)}\left\|\phi_{R} f_{\varepsilon}^{n / 2}\right\|_{2 n /(n-2)}^{2}\right. \\
& \left.-H^{2}\left(1+\frac{1}{t}\right)\left\|\phi_{R} f_{\varepsilon}^{n / 2}\right\|_{2}^{2}\right]+\int_{M} \phi_{R}^{2} f_{\varepsilon}^{n-2} f^{2}\left\{n H^{2}-\left(S-n H^{2}\right)\right. \\
& \left.-\frac{1}{2}\left[\frac{n(n-2)^{2} H^{2}}{l(n-1)}+l\left(S-n H^{2}\right)\right]\right\} d M
\end{aligned}
$$


for all $l \in \mathbf{R}^{+}$. As $\varepsilon \rightarrow 0$, (3.6) becomes

$$
\begin{aligned}
0 \geq & -\left[\frac{4\left(n^{2}-n+2\right)}{n^{3}}-\frac{1}{R}+\frac{R \sigma^{2}}{n^{2}}\right] \int_{M} f^{n}\left|\nabla \phi_{R}\right|^{2} d M \\
& +\frac{\left[4 R\left(n^{2}-n+2\right)-n^{3}\right](n-2)^{2}}{4 R n^{3}(n-1)^{2}(1+t)}\left[\frac{1}{D^{2}(n)}\left\|\phi_{R} f^{n / 2}\right\|_{2 n /(n-2)}^{2}\right. \\
& \left.-H^{2}\left(1+\frac{1}{t}\right)\left\|\phi_{R} f^{n / 2}\right\|_{2}^{2}\right] \\
& +\left[n H^{2}-\frac{n(n-2)^{2} H^{2}}{2 l(n-1)}\right]\left\|\phi_{R} f^{n / 2}\right\|_{2}^{2} \\
& -\left(1+\frac{l}{2}\right)\left\|\phi_{R}^{2} f^{n}\right\|_{n /(n-2)}\left\|S-n H^{2}\right\|_{n / 2} \\
= & -\left[\frac{4\left(n^{2}-n+2\right)}{n^{3}}-\frac{1}{R}+\frac{R \sigma^{2}}{n^{2}}\right] \int_{M} f^{n}\left|\nabla \phi_{R}\right|^{2} d M \\
& +\left\{\frac{\left[4 R\left(n^{2}-n+2\right)-n^{3}\right](n-2)^{2}}{4 R n^{3}(n-1)^{2} D^{2}(n)(1+t)}\right. \\
& \left.-\left(1+\frac{l}{2}\right)\left\|S-n H^{2}\right\|_{n / 2}\right\}\left\|\phi_{R}^{2} f^{n}\right\|_{n /(n-2)} \\
& +\left\{n H^{2}-\frac{n(n-2)^{2} H^{2}}{2 l(n-1)}\right. \\
& \frac{\left[4 R\left(n^{2}-n+2\right)-n^{3}\right](n-2)^{2} H^{2}}{4 R n^{3}(n-1)^{2} t}\left\|\phi_{R} f^{n / 2}\right\|_{2}^{2},
\end{aligned}
$$

where $l \in \mathbf{R}^{+}$. Since $\int_{M} f^{n} d M<\alpha^{n / 2}(n)$, as $R \rightarrow \infty$, we get

$$
\begin{aligned}
0 & \leq \lim _{R \rightarrow \infty}\left[\frac{4\left(n^{2}-n+2\right)}{n^{3}}-\frac{1}{R}+\frac{R \sigma^{2}}{n^{2}}\right] \int_{M} f^{n}\left|\nabla \phi_{R}\right|^{2} d M \\
& \leq \lim _{R \rightarrow \infty}\left[\frac{4\left(n^{2}-n+2\right)}{n^{3}}-\frac{1}{R}+\frac{R \sigma^{2}}{n^{2}}\right] \frac{1}{R^{2}} \int_{M} f^{n} d M=0 .
\end{aligned}
$$


From (3.7) and (3.8), we obtain

$$
\begin{aligned}
0 \geq & {\left[\frac{\left(n^{2}-n+2\right)(n-2)^{2}}{n^{3}(n-1)^{2} D^{2}(n)(1+t)}\right.} \\
& \left.-\left(1+\frac{l}{2}\right)\left\|S-n H^{2}\right\|_{n / 2}\right] \lim _{R \rightarrow \infty}\left\|\phi_{R}^{2} f^{n}\right\|_{n /(n-2)} \\
& +\left[n H^{2}-\frac{n(n-2)^{2} H^{2}}{2 l(n-1)}-\frac{\left(n^{2}-n+2\right)(n-2)^{2} H^{2}}{n^{3}(n-1)^{2} t}\right] \lim _{R \rightarrow \infty}\left\|\phi_{R} f^{n / 2}\right\|_{2}^{2}
\end{aligned}
$$

for all $t, l \in \mathbf{R}^{+}$. We take

$$
t=t(l)=\frac{\left(n^{2}-n+2\right)(n-2)^{2}}{n^{4}(n-1)^{2}}\left[1-\frac{(n-2)^{2}}{2 l(n-1)}\right]^{-1}, \quad l>\frac{(n-2)^{2}}{2(n-1)} .
$$

This together with (3.9) yields

$$
\begin{aligned}
& {\left[\frac{\left(n^{2}-n+2\right)(n-2)^{2}}{n^{3}(n-1)^{2} D^{2}(n)(1+t(l))}\right.} \\
& \left.\quad-\left(1+\frac{l}{2}\right)\left\|S-n H^{2}\right\|_{n / 2}\right] \lim _{R \rightarrow \infty}\left\|\phi_{R}^{2} f^{n}\right\|_{n /(n-2)} \leq 0,
\end{aligned}
$$

where $l \in \mathbf{R}^{+}$satisfying

$$
l>\frac{(n-2)^{2}}{2(n-1)}
$$

By a computation, we have

$$
\max _{l>\frac{(n-2)^{2}}{2(n-1)}} \frac{1}{(2+l)(1+t(l))}=\frac{n^{3}(n-1)^{2} D^{2}(n) \alpha(n)}{2\left(n^{2}-n+2\right)(n-2)^{2}} .
$$

So,

$$
\left[\alpha(n)-\left\|S-n H^{2}\right\|_{n / 2}\right] \lim _{R \rightarrow \infty}\left\|\phi_{R}^{2} f^{n}\right\|_{n /(n-2)} \leq 0 .
$$

From the assumption

$$
\left\|S-n H^{2}\right\|_{n / 2}<\alpha(n),
$$

we conclude that $f \equiv 0$, i.e., $S_{H} \equiv n H^{2}$. Therefore, $M$ is a pseudo-umbilical submanifold. If $p=1$, then $S-n H^{2}=f^{2} \equiv 0$, i.e., $M$ is the totally umbilical sphere $S^{n}(1 / H)$ in $\mathbf{R}^{n+1}$. 
Now we are in a position to give the proof of Theorem 1.4.

Proof of Theorem 1.4. If $H \neq 0$ and $p=1$, the assertion follows from Lemma 3.1.

If $H \neq 0$ and $p \geq 2$, we see from the assumption that $\left\|S-n H^{2}\right\|_{n / 2}<$ $\alpha(n)$. It follows from Lemma 3.1 that $S_{H}=n H^{2}$, i.e., $H_{n+1}=H I$, where $I$ is the unit matrix. By Lemmas 2.2, 2.3 and 2.4, we have

$$
\frac{1}{2} \Delta g_{\varepsilon}^{2}=\frac{1}{2} \Delta g^{2} \geq \frac{n+2}{n}\left|\nabla g_{\varepsilon}\right|^{2}+g^{2}\left[n H^{2}-\mu(p-1) g^{2}\right],
$$

where $g_{\varepsilon}=\left[S_{I}+n(p-1) \varepsilon^{2}\right]^{1 / 2}$ and $g=S_{I}^{1 / 2}$. Multiplying $\phi_{R}^{2} g_{\varepsilon}^{2 k-2}(k \geq 1)$ on both sides of the above inequality and integrating by parts, where $\phi_{R}$ is the cut-off function defined in Lemma 3.1, we obtain

$$
\begin{aligned}
0 \geq & \int_{M} \phi_{R}^{2} g_{\varepsilon}^{2 k-2} g^{2}\left[n H^{2}-\mu(p-1)\left(S-n H^{2}\right)\right] d M \\
& +\frac{n+2}{n k^{2}} \int_{M} \phi_{R}^{2}\left|\nabla g_{\varepsilon}^{k}\right|^{2} d M \\
& +\frac{1}{2} \int_{M} \nabla\left(\phi_{R}^{2} g_{\varepsilon}^{2 k-2}\right) \nabla g_{\varepsilon}^{2} d M-\frac{1}{2} \int_{M} \operatorname{div}\left(\phi_{R}^{2} g_{\varepsilon}^{2 k-2} \nabla g_{\varepsilon}^{2}\right) d M \\
\geq & \int_{M} \phi_{R}^{2} g_{\varepsilon}^{2 k-2} g^{2}\left[n H^{2}-\mu(p-1)\left(S-n H^{2}\right)\right] d M \\
& +\frac{2 n k-n+2}{n k^{2}} \int_{M} \phi_{R}^{2}\left|\nabla g_{\varepsilon}^{k}\right|^{2} d M+2 \int_{M} \phi_{R} g_{\varepsilon}^{2 k-1} \nabla \phi_{R} \nabla g_{\varepsilon} d M \\
& +\sigma \int_{M} \phi_{R} g_{\varepsilon}^{2 k-1} \nabla \phi_{R} \nabla g_{\varepsilon} d M-\frac{\rho \sigma}{2} \int_{M} \phi_{R}^{2} g_{\varepsilon}^{2 k-2}\left|\nabla g_{\varepsilon}\right|^{2} d M \\
& -\frac{\sigma}{2 \rho} \int_{M}\left|\nabla \phi_{R}\right|^{2} g_{\varepsilon}^{2 k} d M
\end{aligned}
$$

for all $\rho, \sigma \in \mathbf{R}^{+}$. Taking $k=\frac{n}{2}, \sigma=\frac{4\left(n^{2}-n+2\right)}{n^{2}}-\frac{n}{R}-2, \rho=\frac{n^{2}}{2 R \sigma}$, where $R>\frac{n^{3}}{2\left(n^{2}-2 n+4\right)}$, we get

$$
\begin{aligned}
0 \geq & -\left[\frac{4\left(n^{2}-n+2\right)}{n^{3}}-\frac{1}{R}+\frac{R \sigma^{2}}{n^{2}}\right] \int_{M} g_{\varepsilon}^{n}\left|\nabla \phi_{R}\right|^{2} d M \\
& +\left[\frac{4\left(n^{2}-n+2\right)}{n^{3}}-\frac{1}{R}\right] \int_{M}\left|\nabla\left(\phi_{R} g_{\varepsilon}^{n / 2}\right)\right|^{2} d M \\
& +\int_{M} \phi_{R}^{2} g_{\varepsilon}^{n-2} g^{2}\left[n H^{2}-\mu(p-1)\left(S-n H^{2}\right)\right] d M .
\end{aligned}
$$


By Lemma 2.5, we have

$$
\begin{aligned}
\left\|\nabla\left(\phi_{R} g_{\varepsilon}^{n / 2}\right)\right\|_{2}^{2} \geq & \frac{(n-2)^{2}}{4(n-1)^{2}(1+t)}\left[\frac{1}{D^{2}(n)}\left\|\phi_{R} g_{\varepsilon}^{n / 2}\right\|_{2 n /(n-2)}^{2}\right. \\
& \left.-H^{2}\left(1+\frac{1}{t}\right)\left\|\phi_{R} g_{\varepsilon}^{n / 2}\right\|_{2}^{2}\right]
\end{aligned}
$$

for all $t \in \mathbf{R}^{+}$. From (3.12) and (3.13), we obtain

$$
\begin{aligned}
0 \geq & -\left[\frac{4\left(n^{2}-n+2\right)}{n^{3}}-\frac{1}{R}+\frac{R \sigma^{2}}{n^{2}}\right] \int_{M} g_{\varepsilon}^{n}\left|\nabla \phi_{R}\right|^{2} d M \\
& +\frac{\left[4 R\left(n^{2}-n+2\right)-n^{3}\right](n-2)^{2}}{4 R n^{3}(n-1)^{2}(1+t)}\left[\frac{1}{D^{2}(n)}\left\|\phi_{R} g_{\varepsilon}^{n / 2}\right\|_{2 n /(n-2)}^{2}\right. \\
& \left.-H^{2}\left(1+\frac{1}{t}\right)\left\|\phi_{R} g_{\varepsilon}^{n / 2}\right\|_{2}^{2}\right] \\
& +\int_{M} \phi_{R}^{2} g_{\varepsilon}^{n-2} g^{2}\left[n H^{2}-\mu(p-1)\left(S-n H^{2}\right)\right] d M .
\end{aligned}
$$

As $\varepsilon \rightarrow 0$, we have

$$
\begin{aligned}
0 \geq & -\left[\frac{4\left(n^{2}-n+2\right)}{n^{3}}-\frac{1}{R}+\frac{R \sigma^{2}}{n^{2}}\right] \int_{M} g^{n}\left|\nabla \phi_{R}\right|^{2} d M \\
& +\frac{\left[4 R\left(n^{2}-n+2\right)-n^{3}\right](n-2)^{2}}{4 R n^{3}(n-1)^{2}(1+t)}\left[\frac{1}{D^{2}(n)}\left\|\phi_{R} g^{n / 2}\right\|_{2 n /(n-2)}^{2}\right. \\
& \left.-H^{2}\left(1+\frac{1}{t}\right)\left\|\phi_{R} g^{n / 2}\right\|_{2}^{2}\right] \\
& +\left[n H^{2}\left\|\phi_{R} g^{n / 2}\right\|_{2}^{2}-\mu(p-1)\left\|S-n H^{2}\right\|_{n / 2}\left\|\phi_{R}^{2} g^{n}\right\|_{n /(n-2)}\right] \\
\geq & -\left[\frac{4\left(n^{2}-n+2\right)}{n^{3}}-\frac{1}{R}+\frac{R \sigma^{2}}{n^{2}}\right] \int_{M} g^{n}\left|\nabla \phi_{R}\right|^{2} d M \\
& +\left\{\frac{\left[4 R\left(n^{2}-n+2\right)-n^{3}\right](n-2)^{2}}{4 R n^{3}(n-1)^{2} D^{2}(n)(1+t)}\right. \\
& \left.-\mu(p-1)\left\|S-n H^{2}\right\|_{n / 2}\right\}\left\|\phi_{R}^{2} g^{n}\right\|_{n /(n-2)} \\
& +\left\{n H^{2}-\frac{\left[4 R\left(n^{2}-n+2\right)-n^{3}\right](n-2)^{2} H^{2}}{4 R n^{3}(n-1)^{2} t}\right\}\left\|\phi_{R} g^{n / 2}\right\|_{2}^{2},
\end{aligned}
$$


where $t \in \mathbf{R}^{+}$. Since $\int_{M} g^{n} d M<C(n)$, as $R \rightarrow \infty$, we get

$$
\begin{aligned}
0 & \leq \lim _{R \rightarrow \infty}\left[\frac{4\left(n^{2}-n+2\right)}{n^{3}}-\frac{1}{R}+\frac{R \sigma^{2}}{n^{2}}\right] \int_{M} g^{n}\left|\nabla \phi_{R}\right|^{2} d M \\
& \leq \lim _{R \rightarrow \infty}\left[\frac{4\left(n^{2}-n+2\right)}{n^{3}}-\frac{1}{R}+\frac{R \sigma^{2}}{n^{2}}\right] \frac{1}{R^{2}} \int_{M} g^{n} d M=0 .
\end{aligned}
$$

As $R \rightarrow \infty,(3.15)$ becomes

$$
\begin{aligned}
0 \geq & {\left[\frac{\left(n^{2}-n+2\right)(n-2)^{2}}{n^{3}(n-1)^{2} D^{2}(n)(1+t)}\right.} \\
& \left.-\mu(p-1)\left\|S-n H^{2}\right\|_{n / 2}\right] \lim _{R \rightarrow \infty}\left\|\phi_{R}^{2} g^{n}\right\|_{n /(n-2)} \\
& +\left[n H^{2}-\frac{\left(n^{2}-n+2\right)(n-2)^{2} H^{2}}{n^{3}(n-1)^{2} t}\right] \lim _{R \rightarrow \infty}\left\|\phi_{R} g^{n / 2}\right\|_{2}^{2}
\end{aligned}
$$

for all $t \in \mathbf{R}^{+}$. By taking $t=\left(n^{2}-n+2\right)(n-2)^{2}\left[n^{4}(n-1)^{2}\right]^{-1}$, we have

$$
\begin{aligned}
& \left\{\frac{n\left(n^{2}-n+2\right)(n-2)^{2}}{D^{2}(n)\left[n^{4}(n-1)^{2}+\left(n^{2}-n+2\right)(n-2)^{2}\right]}-\mu(p-1)\left\|S-n H^{2}\right\|_{n / 2}\right\} \\
& \quad \times \lim _{R \rightarrow \infty}\left\|\phi_{R}^{2} g^{n}\right\|_{n /(n-2)} \leq 0,
\end{aligned}
$$

which implies

$$
\left[\beta(n)-\mu(p-1)\left\|S-n H^{2}\right\|_{n / 2}\right] \lim _{R \rightarrow \infty}\left\|\phi_{R}^{2} g^{n}\right\|_{n /(n-2)} \leq 0 .
$$

It is easy to see from the assumption that

$$
\left\|S-n H^{2}\right\|_{n / 2}<\beta(n) / \mu(p-1) .
$$

This together with (3.18) gives $g \equiv 0$. So,

$$
S-n H^{2}=f^{2}+g^{2} \equiv 0 .
$$

Therefore, $M$ is the totally umbilical sphere $S^{n}(1 / H)$ in $\mathbf{R}^{n+p}$.

If $H=0$, it follows from Lemmas 2.3 and 2.4 that

$$
\frac{1}{2} \Delta h_{\varepsilon}^{2}=\frac{1}{2} \Delta S \geq \frac{n+2}{n}\left|\nabla h_{\varepsilon}\right|^{2}-\mu(p) S^{2},
$$

where $h_{\varepsilon}=\left[S+n p \varepsilon^{2}\right]^{1 / 2}$. Multiplying $\phi_{R}^{2} h_{\varepsilon}^{2 k-2}(k \geq 1)$ on both sides of inequality (3.19) and integrating by parts, where $\phi_{R}$ is the cut-off function 
defined in Lemma 3.1, we obtain

$$
\begin{aligned}
0 \geq & -\mu(p) \int_{M} \phi_{R}^{2} h_{\varepsilon}^{2 k-2} S^{2} d M+\frac{n+2}{n k^{2}} \int_{M} \phi_{R}^{2}\left|\nabla h_{\varepsilon}^{k}\right|^{2} d M \\
& +\frac{1}{2} \int_{M} \nabla\left(\phi_{R}^{2} h_{\varepsilon}^{2 k-2}\right) \nabla h_{\varepsilon}^{2} d M-\frac{1}{2} \int_{M} \operatorname{div}\left(\phi_{R}^{2} h_{\varepsilon}^{2 k-2} \nabla h_{\varepsilon}^{2}\right) d M \\
\geq & -\mu(p) \int_{M} \phi_{R}^{2} h_{\varepsilon}^{2 k-2} S^{2} d M+\frac{2 n k-n+2}{n k^{2}} \int_{M} \phi_{R}^{2}\left|\nabla h_{\varepsilon}^{k}\right|^{2} d M \\
& +2 \int_{M} \phi_{R} h_{\varepsilon}^{2 k-1} \nabla \phi_{R} \nabla h_{\varepsilon} d M \\
& +\sigma \int_{M} \phi_{R} h_{\varepsilon}^{2 k-1} \nabla \phi_{R} \nabla h_{\varepsilon} d M-\frac{\rho \sigma}{2} \int_{M} \phi_{R}^{2} h_{\varepsilon}^{2 k-2}\left|\nabla h_{\varepsilon}\right|^{2} d M \\
& -\frac{\sigma}{2 \rho} \int_{M}\left|\nabla \phi_{R}\right|^{2} h_{\varepsilon}^{2 k} d M
\end{aligned}
$$

for all $\rho, \sigma \in \mathbf{R}^{+}$. Taking $k=\frac{n}{2}, \sigma=\frac{4\left(n^{2}-n+2\right)}{n^{2}}-\frac{n}{R}-2, \rho=\frac{n^{2}}{2 R \sigma}$, where $R>\frac{n^{3}}{2\left(n^{2}-2 n+4\right)}$, we get

$$
\begin{aligned}
0 \geq & -\left[\frac{4\left(n^{2}-n+2\right)}{n^{3}}-\frac{1}{R}+\frac{R \sigma^{2}}{n^{2}}\right] \int_{M} h_{\varepsilon}^{n}\left|\nabla \phi_{R}\right|^{2} d M \\
& +\left[\frac{4\left(n^{2}-n+2\right)}{n^{3}}-\frac{1}{R}\right] \int_{M}\left|\nabla\left(\phi_{R} h_{\varepsilon}^{n / 2}\right)\right|^{2} d M \\
& -\mu(p) \int_{M} \phi_{R}^{2} h_{\varepsilon}^{n-2} S^{2} d M .
\end{aligned}
$$

By Lemma 2.5, we have

$$
\left\|\nabla\left(\phi_{R} h_{\varepsilon}^{n / 2}\right)\right\|_{2}^{2} \geq \frac{(n-2)^{2}}{4(n-1)^{2} D^{2}(n)}\left\|\phi_{R} h_{\varepsilon}^{n / 2}\right\|_{2 n /(n-2)}^{2} .
$$

From (3.21) and (3.22), we obtain

$$
\begin{aligned}
0 \geq & -\left[\frac{4\left(n^{2}-n+2\right)}{n^{3}}-\frac{1}{R}+\frac{R \sigma^{2}}{n^{2}}\right] \int_{M} h_{\varepsilon}^{n}\left|\nabla \phi_{R}\right|^{2} d M \\
& +\frac{\left[4 R\left(n^{2}-n+2\right)-n^{3}\right](n-2)^{2}}{4 R n^{3}(n-1)^{2} D^{2}(n)}\left\|\phi_{R} h_{\varepsilon}^{n / 2}\right\|_{2 n /(n-2)}^{2} \\
& -\mu(p) \int_{M} \phi_{R}^{2} h_{\varepsilon}^{n-2} S^{2} d M .
\end{aligned}
$$


As $\varepsilon \rightarrow 0$, this implies

$$
\begin{aligned}
0 \geq & -\left[\frac{4\left(n^{2}-n+2\right)}{n^{3}}-\frac{1}{R}+\frac{R \sigma^{2}}{n^{2}}\right] \int_{M} h^{n}\left|\nabla \phi_{R}\right|^{2} d M \\
& +\frac{\left[4 R\left(n^{2}-n+2\right)-n^{3}\right](n-2)^{2}}{4 R n^{3}(n-1)^{2} D^{2}(n)}\left\|\phi_{R}^{2} S^{n / 2}\right\|_{n /(n-2)} \\
& -\mu(p) \int_{M} \phi_{R}^{2} S^{n / 2+1} d M \\
\geq & -\left[\frac{4\left(n^{2}-n+2\right)}{n^{3}}-\frac{1}{R}+\frac{R \sigma^{2}}{n^{2}}\right] \int_{M} h^{n}\left|\nabla \phi_{R}\right|^{2} d M \\
& +\frac{\left[4 R\left(n^{2}-n+2\right)-n^{3}\right](n-2)^{2}}{4 R n^{3}(n-1)^{2} D^{2}(n)}\left\|\phi_{R}^{2} S^{n / 2}\right\|_{n /(n-2)} \\
& -\mu(p)\left\|\phi_{R}^{2} S^{n / 2}\right\|_{n /(n-2)}\|S\|_{n / 2} .
\end{aligned}
$$

Since

$$
\lim _{R \rightarrow \infty}\left[\frac{4\left(n^{2}-n+2\right)}{n^{3}}-\frac{1}{R}+\frac{R \sigma^{2}}{n^{2}}\right] \int_{M} h^{n}\left|\nabla \phi_{R}\right|^{2} d M=0
$$

as $R \rightarrow \infty,(3.24)$ becomes

$$
\left[\gamma(n)-\mu(p)\|S\|_{n / 2}\right] \lim _{R \rightarrow \infty}\left\|\phi_{R}^{2} S^{n / 2}\right\|_{n /(n-2)} \leq 0 .
$$

We see from the assumption that $\|S\|_{n / 2}<\gamma(n) / \mu(p)$. This together with (3.25) implies $S \equiv 0$, i.e., $M$ is the totally geodesic submanifold $\mathbf{R}^{n}$ in $\mathbf{R}^{n+p}$. This completes the proof of Theorem 1.4.

Remark 3.2. We see from the proof above that the pinching constant in Theorem 1.4 can be replaced by the constant $B^{n / 2}(n, p, H)$ defined in (3.1), which is not less than $C(n)$.

When the ambient space is a sphere with positive constant curvature $c$, we have the following lemma.

Lemma 3.3. Let $M^{n}(n \geq 3)$ be an $n$-dimensional complete submanifold with parallel mean curvature in an $(n+p)$-dimensional sphere $S^{n+p}\left(\frac{1}{\sqrt{c}}\right)$, where $c$ is a positive constant. Denote by $H$ and $S$ the mean curvature and the squared length of the second fundamental form of $M$, respectively. If $\int_{M}\left(S-n H^{2}\right)^{n / 2} d M<C(n)$, where $C(n)$ is an explicit positive constant depending only on $n$, then $M$ is the totally umbilical sphere $S^{n}\left(\frac{1}{\sqrt{c+H^{2}}}\right)$. 
Proof. It follows from the assumption that $S^{n+p}\left(\frac{1}{\sqrt{c}}\right)=\mathbf{F}^{n+p}(c)$. We consider the composition of isometric immersions

$$
i \circ \varphi: M^{n} \rightarrow \mathbf{F}^{n+p}(c) \rightarrow \mathbf{R}^{n+p+1},
$$

where $\varphi: M^{n} \rightarrow \mathbf{F}^{n+p}(c)$ is the isometric immersion, and $i$ is the standard isometric embedding of $\mathbf{F}^{n+p}(c)$ into $\mathbf{R}^{n+p+1}$. Denote by $\widetilde{H}$ and $\widetilde{S}$ the mean curvature and the squared length of the second fundamental form of the isometric immersion $i \circ \varphi$, respectively. Then $i \circ \varphi(M)$ is a complete submanifold in $\mathbf{R}^{n+p+1}$ with parallel mean curvature vector having norm $\widetilde{H}$. By the Gauss equation, we have

$$
n(n-1) c+n^{2} H^{2}-S=n^{2} \widetilde{H}^{2}-\widetilde{S} .
$$

Substituting $\widetilde{H}^{2}=c+H^{2}$ into the above, we get $S-n H^{2}=\widetilde{S}-n \widetilde{H}^{2}$. The claim follows directly from Theorem 1.4.

Proof of Theorem 1.6. Combining Theorem 1.4 and Lemma 3.3, we complete the proof of Theorem 1.6.

Remark 3.4. By using the same argument as in Theorem 1.4, one can obtain an analogous gap theorem for complete submanifold with parallel mean curvature in hyperbolic space $\mathbf{H}^{n+p}(-1)$.

\section{Acknowledgement}

The authors would like to thank Professors Kefeng Liu and Shing-Tung Yau for their constant encouragement and valuable suggestions. Thanks also to Professor Chunli Shen for helpful discussions. Research supported by the Chinese NSF, Grant No. 10231010; the Trans-Century Training Program Foundation for Talents by the Ministry of Education of China; and the Natural Science Foundation of Zhejiang Province, Grant No. 101037.

\section{References}

[1] H. Alencar and M. do Carmo, Hypersurfaces with constant mean curvature in sphere, Proc. Am. Math. Soc. 120 (1994), 1223-1229.

[2] S. S. Chern, M. do Carmo, and S. Kobayashi, Minimal submanifolds of a sphere with second fundamental form of constant length, Functional Analysis and Related Fields, Springer-Verlag, 1970, pp. 59-75. 
[3] Z. Guo, H. Z. Li, and C. P. Wang, The second variation formula for Willmore submanifolds in $S^{n}$, Results Math. 40 (2001), 205-225.

[4] D. Hoffman and J. Spruck, Sobolev and isoperimetric inequalities for Riemannian submanifolds, Commun. Pure Appl. Math. 27 (1974), 715727.

[5] H. B. Lawson, Local rigidity theorems for minimal hyperfaces, Ann. Math. 89 (1969), 187-197.

[6] A. M. Li and J. M. Li, An intrinsic rigidity theorem for minimal submanifold in a sphere, Arch. Math. 58 (1992), 582-594.

[7] P. Li, On the Sobolev constant and the p-spectrum of a compact Riemannian manifold, Ann. Sci. Ec. Norm. Sup. 4e serie, t. 13 (1980), 451-469.

[8] J. M. Lin and C. Y. Xia, Global pinching theorem for even dimensional minimal submanifolds in a unit sphere, Math. Z. 201 (1989), 381-389.

[9] L. Ni, Gap theorems for minimal submanifolds in $\mathbf{R}^{n+1}$, Commun. Anal. Geom. 9 (2001), 641-656.

[10] M. Okumura, Hypersurfaces and a pinching problem on the second fundamental tensor, Am. J. Math. 96 (1974), 207-213.

[11] C. L. Shen, A global pinching theorem for minimal hypersurfaces in sphere, Proc. Am. Math. Soc. 105 (1989), 192-198.

[12] J. Simons, Minimal varieties in Riemannian submanifolds, Ann. Math. 88 (1968), 62-105.

[13] K. Shiohama and H. W. Xu, A general rigidity theorem for complete submanifolds, Nagoya Math. J. 150 (1998), 105-134.

[14] K. Shiohama and H. W. Xu, The topological sphere theorem for complete submanifolds, Compositio Math. 107 (1997), 221-232.

[15] R. Schoen and S. T. Yau, Differential geometry, Academic Press, Beijing, 1988.

[16] H. Wang, Some global pinching theorems for minimal submanifolds in a sphere, Acta. Math. Sin. 31 (1988), 503-509.

[17] H. W. Xu, A pinching constant of Simons' type and isometric immersion, Chin. Ann. Math. 12 (1991), 261-269. 
[18] H. W. Xu, A rigidity theorem for submanifold with parallel mean curvature in a sphere, Arch. Math. 61 (1993), 489-496.

[19] H. W. Xu, Pinching theorems, global pinching theorems and eigenvalues for Riemannian submanifolds, Ph.D. dissertation, Fudan University, 1990.

[20] H. W. Xu, $L_{n / 2}$-pinching theorems for submanifolds with parallel mean curvature in a sphere, J. Math. Soc. Japan 46 (1994), 503-515.

[21] H. W. Xu, On closed minimal submanifolds in pinched Riemannian manifolds, Trans. Am. Math. Soc. 347 (1995), 1743-1751.

[22] S. T. Yau, Submanifolds with constant mean curvature I, II, Am. J. Math. 96 (1974), 346-366; 97 (1975), 76-100.

Center of Mathematical Sciences

ZHEJIANG UNIVERSITY

HANGZHOU 310027

CHINA

E-mail address: xuhw@cms.zju.edu.cn

E-mail address: gujuanru@sina.com.cn

Received February 2, 2006 
